\title{
Addressing the vaccination gap in eldercare
}

\author{
Vaccine hesitancy has created a gap in COVID-19 vaccination status between residents and staff in long-term \\ care settings. Closing that gap is essential to protect our most vulnerable populations and fulfill the duty of care \\ they deserve.
}

T he roll-out earlier this year of age-based COVID-19 vaccination schedules around the world made a lot of sense. Overwhelming evidence had pointed to age as a major risk factor for developing severe COVID-19 disease. Besides the age-based vaccination schedule, priority was also given to long-term care facilities (LTCFs). LTCFs are congregate settings, and their residents are highly vulnerable to COVID-19 disease; it is estimated that they account for $\sim 40 \%$ of all COVID-19-related deaths. However, COVID-19 vaccine clinical trials were not powered to draw robust conclusions about efficacy in age subgroups - in particular, adults over the age of 70 - and the trial cohorts were healthier than LTCF residents who tend to have multiple comorbidities. As comorbidities substantially increase the risk of severe COVID-19 illness and older adults are less likely to develop protective immunity following immunizations, uncertainty remained about vaccine efficacy in the most vulnerable populations, particularly those who live in long-term care settings. Encouraging real-world data on the effectiveness of vaccines in nursing homes is starting to emerge ${ }^{1}$, and national statistics show vaccination campaigns have reduced the number of COVID-19-related deaths in those facilities.

Some of the vaccination campaigns have successfully brought the vaccine to LTCFs and immunized those at the highest risk (the residents), but much less success has commonly been observed for LTCF staff with equal access to immunization. While rates of vaccination are usually high for residents $(>80 \%)$, those numbers can fall sharply $^{2}$ to $50 \%$ or less for staff. This means the virus can still make its way into care facilities. In one recent example ${ }^{3}$, infection of a skilled nursing facility staff member in the USA who was not vaccinated led to the infection of a large fraction of residents and healthcare workers in that facility, both vaccinated and unvaccinated. Although infection and disease severity were lower in vaccinated individuals during this outbreak, several residents died or were hospitalized. Some of these deaths could have been prevented if higher rates of vaccination had been achieved, in particular, among healthcare workers, which is a reminder that efforts must continue to promote vaccination in those settings.

The vaccination gap that exists between residents and care workers is not a new problem; a similar gap exists for flu vaccination. One of the main reasons behind COVID-19 vaccine hesitancy in LTCF staff is concern about side effects, including the remote possibility of long-term health side effects ${ }^{4}$. Lack of trust is also a contributing factor, possibly fueled by misinformation. Other reasons may be more practical. The known short-term side effects of the vaccine, such as fatigue, may impact one's ability to work and earn an income. LTCF personnel tend to earn lower wages than other healthcare professionals and often complement their income by having more than one job, sometimes with no prospect of sick pay. Some of these issues are being addressed by informing the staff of the costto-benefit ratio of vaccination for themselves, and the benefit of their own vaccination for those they care for, but more could be done: for instance, by implementing specific sickpay schemes to help maintain income in case of side effects. Other concerns may also be at play, and surveying staff for the reasons behind their reluctance to get vaccinated and addressing these concerns is an important step to getting people on board.

The vaccination gap poses an important challenge to long-term care institutions as it challenges their ability to fulfill their duty of care. To address the issue, some have chosen to impose vaccination as a condition for employment. Although vaccine mandates remain controversial, various other institutions in many countries, private or public, such as schools and hospitals, have previously mandated vaccination against infectious diseases like the flu or measles. The motivation behind these mandates is to protect populations, and, in particular, populations at risk. In that spirit, it does make sense to mandate COVID-19 vaccines in LTCFs. But imposing vaccination mandates may have unintended consequences, as workers presented with the choice to accept vaccination or leave and work elsewhere may well choose the latter. There is already a severe shortage of healthcare professionals willing to work in long-term care settings, and many fear that enforcing vaccination mandates risks accentuating the problem and diminishing the quality of care that can be offered.

Various experts anticipate that SARS$\mathrm{CoV}-2$ will become endemic like seasonal $\mathrm{flu}^{5,6}$. The existence and emergence of more highly transmissible virus variants and the current lack of clarity on the duration of protective immunity post-immunization point to the need for a long-term vaccination agenda to efficiently protect the most vulnerable. As the deployment of mandates is being considered, approaches that take into account the multiple sociocultural and scientific dimensions of the problem and rely on evidence are needed. This will require government support of efforts to continue the collection of real-world data on vaccine effectiveness and rates in different settings, and to estimate the cost in lives and healthcare expenditure where mandates are in effect or not. Some of these efforts are underway. The US government recently instituted weekly reports on COVID-19 vaccination status for both staff and residents in LTCFs. In the UK, the government has launched a national consultation on COVID-19 vaccination mandates in care homes. A multi-tier approach to mandates could be considered, as the best solutions may depend on the type of facility or other situational factors. Imposing strict mandates in places hosting the most vulnerable individuals, such as skilled nursing homes, for instance, may be necessary, but implementing solutions that also take into consideration the needs of staff is essential. Importantly, transparent sharing of information with existing and prospective residents and their families about the risks in different facilities should also be imposed by regulators.

Addressing the vaccination gap to achieve maximum COVID-19 immunity in long-term care settings is a prerequisite to adequately fulfilling their duty of care. To achieve this goal, the implementation of tailored vaccine mandates, alongside sustainable support schemes that better value LTCF workers, seems inevitable.

Published online: 14 June 2021 https://doi.org/10.1038/s43587-021-00081-z 
References

1. White, E. M. et al. New Engl. J. Med. https://doi.org/10.1056/ NEJMc2104849 (2021)
2. Gharpure, R. et al. Morbid. Mortal. W. 70, 178-182 (2021).

3. Cavanaugh, A. M. et al. Morbid. Mortal. W. 70, 639-643 (2021).

4. Unroe, K. T. et al. J. Am. Geriatr. Soc. 69, 593-599 (2021).
5. Murray, C. J. L. \& Piot, P. J. Am. Med. Assoc. 325 $1249-1250$ (2021).

6. Phillips, N. Nature 590, 382-384 (2021). 